\title{
On Solutions of Emden-Fowler Equation
}

\author{
Khalid A. A. Hossen \\ Department of Computer Science, Faculty of Information Technology, Al-Ahliyya Amman University, Amman, Jordan \\ Email: kaljibouri@ammanu.edu.jo
}

How to cite this paper: Hossen, K.A.A. (2020) On Solutions of Emden-Fowler Equation. American Journal of Computational Mathematics, 10, 90-99. https://doi.org/10.4236/ajcm.2020.101006

Received: August 21, 2019

Accepted: March 8, 2020

Published: March 11, 2020

Copyright $\odot 2020$ by author(s) and Scientific Research Publishing Inc. This work is licensed under the Creative Commons Attribution International License (CC BY 4.0).

http://creativecommons.org/licenses/by/4.0/

\begin{abstract}
Finite Element Method (FEM), based on $\mathrm{p}$ and $\mathrm{h}$ versions approach, and the Adomians decomposition algorithm (ADM) are introduced for solving the Emden-Fowler Equation. A number of special cases of $\mathrm{p}$ and $\mathrm{h}$ versions of FEM are introduced. Several iterated forms of the ADM are considered also. To demonstrate the efficiency of both methods, the numerical solutions of different examples are compared for both methods with the analytical solutions. It is observed that the results obtained by FEM are quite satisfactory and more accurate than ADM. Moreover, the FEM method is applicable for a wide range of classes including the singularity cases with the given special treatments by the FEM. Comparing the results with the existing true solutions shows that the FEM approach is highly accurate and converges rapidly.
\end{abstract}

\section{Keywords}

Finite Element Method, Adomians Decomposition Algorithm, Numerical, Error

\section{Introduction}

In this work, we present an alternate algorithm to solve the Emden-Fowler Equation [1] [2]. This equation has several interesting physical applications occurring in astrophysics in the form of the Fermi-Thomas equation [3]. The analysis is accompanied by examples that demonstrate the comparison and show the pertinent features of the modified technique. Two versions of FEM approaches have been used to obtain a numerical solution to this problem. The decomposition scheme representing the nonlinear problem is presented. Some references for such numerical solutions can be found in [4] [5] [6]. In particular, Scott [7] used an invariant imbedding method to solve Troesch's problem, while Khuri [8] used a numerical method based on Laplace transformation and a modified decomposition technique to obtain an approximate solution of the same prob- 
lem. Feng [9] solved this problem numerically using a modified homotopy perturbation technique. Chang and Chang [10] developed a new technique for calculating the one-dimensional differential transform of nonlinear functions; the algorithm was illustrated by studying several nonlinear ordinary differential equations, including Troesch's problem. Chang [11] proposed a new algorithm based on the vibrational method and variable transformation to solve Troesch's problem. The cubic B-spline finite-element method (see [12] [13] [14]) is often used for solving nonlinear problems that arise in engineering applications; cubic B-spline functions are utilized to develop a collocation method for solving Troesch's problem.

Adomians decomposition algorithm has been recently employed to solve a wide range of problems (see [15] [16] [17]). We adapt the algorithm to solving the most general form of Emden-Fowler Equation given by

$$
\frac{\mathrm{d}}{\mathrm{d} t}\left(q(t) \frac{\mathrm{d} u}{\mathrm{~d} t}\right)+q(t) u^{n}=0, t \geq 0
$$

with specified initial condition

$$
\begin{gathered}
u(0)=\alpha \\
\frac{\mathrm{d} u}{\mathrm{~d} t}=\beta
\end{gathered}
$$

The balance of this paper is as follows. In Section 2.1, we give a brief description of Adomians method and then introduce a modified version of this algorithm. We apply the modified scheme to Equations (1), (2). While in Section 2.2, we seek a finite element solution for solving Emden-Fowler Equation. We consider the mesh point $u_{i}$ over the interval $[0,1]$, with $x_{0}=0$ and $x_{n}=1$, noting that the mesh points distance $=h$.

In Section 3, several interesting examples that arise in applications are used to illustrate the algorithm with error estimates.

And tables are not prescribed, although the various table text styles are provided. The formatter will need to create these components, incorporating the applicable criteria that follow.

\section{Analysis}

\subsection{Adomians Decomposition Method}

In this section, we first describe algorithm of Adomians decomposition method as it applies to general nonlinear equation of the form

$$
u-N(u)=f
$$

where $N$ is a nonlinear operator on a Hilbert space $H$ and $f$ is a known element of $H$. We assume that for a given $\mathrm{f}$ a unique solution $u$ of Equation (3) exists. We then introduce a modified version of this algorithm to handle equations of the form Equation (1).

The Adomians algorithm assumes a series solution for $u$ given by

$$
u=\sum_{n=0}^{\infty} u_{n}
$$


And the nonlinear operator $\mathrm{N}$ to be decomposed into

$$
N(u)=\sum_{n=0}^{\infty} A_{n}
$$

where $A_{n}$ 's are Adomians polynomials of $u_{0}, u_{1}, \cdots, u_{n}$ given by

$$
A_{n}=\frac{1}{n !} \frac{\mathrm{d}^{n}}{\mathrm{~d} \lambda^{n}}\left[N \sum_{i=0}^{\infty} \lambda^{i} u_{i}\right]_{\lambda=0}, n=0,1, \cdots
$$

Substituting Equations (4) and (5) into the functional Equation (3) yields.

$$
\sum_{n=0}^{\infty} u_{n}-\sum_{n=0}^{\infty} A_{n}=f
$$

The convergence of the series in Equation (7) will yield

$$
\begin{aligned}
u_{0} & =f \\
u_{1} & =A_{0} \\
u_{2} & =A_{1} \\
& \vdots \\
u_{n} & =A_{n-1}
\end{aligned}
$$

Thus, one can, recurrently determine every term of the series $\sum_{n=0}^{\infty} u_{n}$ the convergence of this series has been established (see [17] [18]).

To illustrate the scheme, let the nonlinear operator $N(u)$ be a nonlinear function of $u$, say $g(u)$ then the first four Adomians Polynomials are given by

$$
\begin{aligned}
A_{0} & =g\left(u_{0}\right) \\
A_{1} & =u_{1} g^{\prime}\left(u_{0}\right) \\
A_{2} & =u_{2} g^{\prime}\left(u_{0}\right)+\frac{1}{2 !} u_{1}^{2} g^{\prime \prime}\left(u_{0}\right) \\
& \vdots \\
A_{3} & =u_{3} g^{\prime}\left(u_{0}\right)+u_{1} u_{2} g^{\prime \prime}\left(u_{0}\right)+\frac{1}{3 !} u_{1}^{3}+g^{\prime \prime \prime}\left(u_{0}\right)
\end{aligned}
$$

How do we interpret and solve Emden-Fowler Equation in this setting?

Following the Adomians decomposition analysis [4] defines the linear operator.

$$
L_{t}=\frac{\partial}{\partial t}
$$

Equation (1) can be rewritten in terms of the linear operator

$$
L_{t}\left[p(t) L_{t}(u)\right]+q(t) u_{n}=0
$$

It was shown in [2] that Equation (1) with condition (2) possesses a unique solution.

Thus, the inverse operator of $L_{t}$, namely $L_{t}^{-1}$ exists and is the twofold indefinite integral; i.e.

$$
\left[L_{t}^{-1} f\right](t)=\int_{0}^{t} \mathrm{~d} u \int_{0}^{t} \mathrm{~d} v f(v)
$$

Operating on both sides of (11) with $L_{t}^{-1}$ yields

$$
L_{t}^{-1} L_{t}\left[p(t) L_{t}(u)\right]+L_{t}^{-1}\left[q(t) u^{n}\right]=0
$$


From which it follows, upon using the initial conditions given in Equation (2).

$$
u(t)=u(0)+L_{t}^{-1}\left[\frac{p(0) \beta}{p(t)}\right]-L_{t}^{-1}\left[\frac{L_{t}^{-1}\left(q(t) u^{n}\right)}{P(t)}\right]
$$

The Adomians decomposition method yields the solution in the form given in (4) i.e. $u(t)=u_{0}+u_{1}+u_{2}+\cdots+u_{n}$, then from Equation (14)

$$
u_{0}+u_{1}+u_{2}+\cdots=1+L_{t}^{-1}\left[\frac{p(0) \beta}{\beta(t)}\right]-L_{t}^{-1}\left[\frac{L_{t}^{-1}\left(q(t) u^{n}\right)}{p(t)}\right]
$$

For later numerical computation, let the expression

$$
\phi_{n}=\sum_{i=0}^{n-1} u_{i}(t)
$$

can serve as a practical solution. We will show through several examples, that this Adomians decomposition method which converts the given equation to recurrences relation whose terms are computed using maple 15.

\subsection{Finite Element Method (FEM)}

We seek a finite element solution for solving Emden-Fowler Equation, we consider the mesh point $u_{i}$ over the interval $[0,1]$, with $x_{0}=0$ and $x_{n}=1$, note that the mesh points distance $=h$, with $x_{n}=x_{0}+i h$. Given

$$
u(x)=\sum_{n=0}^{n-1} \alpha_{i} \varphi_{i}
$$

The finite element method minimizes the integral (17) over a class of Piecewise polynomials. The idea is to choose a finite number of trial functions $\varphi_{i}$, and among all their linear combinations $\sum_{n=0}^{n-1} \alpha_{i} \varphi_{i}$ to find the one, which is the minimum, the unknown $\alpha_{i}$ determined by a system of $\mathrm{N}$ discrete algebraic equations, which the computer can handle. Therefore, the goal is to choose trial functions $\varphi_{i}$, which are convenient enough for the given integral (17) to be compute and minimized, and at the same time general enough to approximate closely the unknown $u$. The software TWEPEP starts by a subdivision of the given region into smaller pieces which are triangles with standard six-node with a quadratic basis function, and with one edge curved when adjacent to a curved boundary according to the isoperimetric method. It is also optional to use 10-piont cubic (3rd degree) or the 15-points quartic (4th degree) isoperimetric triangular elements for greater accuracy. Each time a triangle is partitioned, it is divided by a line from the midpoint of its longest side to the opposite vertex. If this side is not on the boundary, the triangle which shares that side must also be divided to avoid non-conforming elements with discontinuous basis functions. An initial triangulation with sufficient triangles to define the region is supplied by the user, then the refinement and grading of this triangulation is guided by a user supplied function D3EST which should be largest where the final triangulation is to be densest. The Cuthill-McKee algorithm ([18] [19] [20]) used to initially number the nodes, and a special bandwidth reduction algorithm is used to 
decrease the bandwidth of the Jacobian matrix even further.

In all cases, the algebraic system solved by Newton's method. One iteration per time step is done for parabolic problems and one iteration is sufficient for linear elliptic problems. The linear system is solved directly by block Gaussian elimination, without row interchanges since pivoting is unnecessary when the matrix is positive definite. Symmetry also taken advantage of in the elimination process if it is present then the storage and computational work halved. If the Jacobian matrix is too large to keep in core, the frontal method is used efficiently organize its storage out of core.

\section{Illustrations of the Methods}

In this section, we shall consider three examples the first is the linearized Emden-Fowler Equation, and the other two are nonlinear. The out-come of modified Adomians decomposition method compared with and the finite element method the $\mathrm{p}$, the $\mathrm{h}$ versions, and any known solution to the underlying Emden-Fowler Equation. Then solution obtained generalized by maple [21]. While the nonlinear system of equations given in (17) has been solved using the old version of the computer algebra system TWEPEP [22].

Example 1. Consider Emden-Fowler Equation of the form

$$
\begin{aligned}
& \frac{1}{t^{2}} \frac{\mathrm{d}}{\mathrm{d} t}\left(t^{2} \frac{\mathrm{d}}{\mathrm{d} t}(u)\right)+t^{2} u=0 \\
& u(0)=1 \text { and } \frac{\mathrm{d} u(0)}{\mathrm{d} t}=0
\end{aligned}
$$

Substituting these values into the general formula Equation (13), we obtain

$$
\begin{aligned}
& u_{0}=1 \\
& u_{1}=-L_{t}^{-1}\left(\frac{t^{2} u_{0}}{t^{2}}\right)=-\frac{1}{6} t^{2} \\
& u_{2}=-L_{t}^{-1}\left(\frac{t^{2} u_{1}}{t^{2}}\right)=\frac{1}{120} t^{4} \\
& u_{3}=-L_{t}^{-1}\left(\frac{t^{2} u_{2}}{t^{2}}\right)=-\frac{1}{5040} t^{6}
\end{aligned}
$$

The exact solution to Equation (18) given by $u(t)=\frac{1}{t} \sin t$.

Example 2. Consider the Emden Fowler of the form

$$
\begin{gathered}
\frac{1}{t^{2}}\left(\frac{\mathrm{d}}{\mathrm{d} t}\left(t^{2} \frac{\mathrm{d}}{\mathrm{d} t} u\right)\right)+u^{5}=0 \\
u(0)=1, \frac{\mathrm{d} u(0)}{\mathrm{d} t}=0
\end{gathered}
$$

Using Equation (13), the various $u_{i}$ are given by 


$$
\begin{aligned}
& u_{0}=1 \\
& u_{1}=-L_{t}^{-1}\left(\frac{t^{2} A_{0}}{t^{2}}\right)=-\frac{1}{6} t^{2} \\
& u_{2}=-L_{t}^{-1}\left(\frac{t^{2} A_{1}}{t^{2}}\right)=-\frac{1}{24} t^{4} \\
& u_{3}=-L_{t}^{-1}\left(\frac{t^{2} A_{2}}{t^{2}}\right)=-\frac{5}{432} t^{6}
\end{aligned}
$$

where $A_{0}=f\left(u_{0}\right)=1$

$$
\begin{gathered}
A_{1}=u_{1} f^{\prime}\left(u_{0}\right)=5 u_{0}^{5}=-\frac{5}{6} t^{2} \\
A_{2}=u_{2} f^{\prime}\left(u_{0}\right)+\frac{1}{2} u_{1}^{2} f^{\prime \prime}\left(u_{0}\right)=5 u_{0}^{4} u_{2}+10 u_{0}^{3} u_{1}^{2}=\frac{35}{71} t^{4} \\
A_{3}=u_{3} f^{\prime}\left(u_{0}\right)+\frac{1}{2}\left(2 u_{1} u_{2}\right) f^{\prime \prime}\left(u_{0}\right)+\frac{1}{3 !}\left(u_{0} u_{1}^{3}\right) f^{\prime \prime \prime}\left(u_{0}\right)=-\frac{35}{144} t^{6}
\end{gathered}
$$

The exact solution of Equation (20) is given by

$$
u(t)=\frac{1}{\sqrt{1+\frac{1}{3} t}}
$$

The exact solution compared with the numerical solution using Adomians method.

Example 3. Our final Example deals with Emden-Fowler Equation of the form

$$
\begin{gathered}
\frac{\mathrm{d}}{\mathrm{d} t}\left(\frac{1}{\sqrt{1+t}}\left(\frac{\mathrm{d}}{\mathrm{d} t}(u)\right)\right)+\frac{1}{2(1+t)^{3}} u^{2}=0 \\
u(0)=1, \frac{\mathrm{d} u(0)}{\mathrm{d} t}=\frac{1}{2}
\end{gathered}
$$

Using Equation (13) the various iterates are given by

$$
\begin{aligned}
& u_{0}=1+\frac{1}{2} L_{t}^{-1}\left[L_{t}^{-1}[1+t]^{1 / 2}\right]=\frac{2}{3}+\frac{1}{3}(1+t)^{3 / 2} \\
& u_{1}=-\frac{1}{2} L_{t}^{-1}\left[L_{t}^{-1}\left(\frac{A_{0}}{(1+t)^{3}}\right)(1+t)^{1 / 2}\right] \\
& u_{2}=-\frac{1}{2} L_{t}^{-1}\left[L_{t}^{-1}\left(\frac{A_{1}}{(1+t)^{3}}\right)(1+t)^{1 / 2}\right] \\
& u_{3}=-\frac{1}{2} L_{t}^{-1}\left[L_{t}^{-1}\left(\frac{A_{2}}{(1+t)^{3}}\right)(1+t)^{1 / 2}\right]
\end{aligned}
$$

where

$$
A_{0}=f\left(u_{0}\right)=u_{0}^{2}=\left[\frac{2}{3}+\frac{1}{3}(1+t)^{3 / 2}\right]^{2}
$$




$$
\begin{aligned}
& A_{1}=2 u_{0} u_{1} \\
& A_{2}=2 u_{0} u_{1}+u_{1}^{2} \\
& A_{3}=2 u_{0} u_{3}+2 u_{0} u_{1} u_{2}
\end{aligned}
$$

:

The exact solution of Equation (23) and (24) is given by

$$
u(t)=\sqrt{1+t}
$$

The exact solution compared with the numerical solution using Adomians method, shown in Table 3 errors obtained by approximation $\phi_{4}, \phi_{5}$ and $\phi_{6}$ as defined in Equation (16) respectively.

In the next section, we will give the numerical results arising from the implementation of this adaptive collocation approach over the Emden-Fowler problem.

In this section, the ADM collocation method used to solve the Emden-Fowler problem for different values of the $u_{i}$ using the computer algebra system Maple 15. In Tables 1-3, the numerical solutions obtained by the above method at the mesh points $t=0,0.01,0.02, \cdots, 0.1$. Respectively, are compared with the given analytic solutions the resulting relative error is also compared with $h$ version FEM numerical techniques, namely, 50, 100 and 150 elements.

In Tables 4-6, the numerical solution obtained by the FEM collocation at the mesh points $t=0,0.01,0.02, \cdots, 0.1$, is compared with the exact solutions given. A Fortran code called TWOPEP [22] used to solve to solve the problems.

Table 1. Error obtained using decomposition method for example 1.

\begin{tabular}{ccccccc}
\hline \multirow{2}{*}{$\mathrm{t}$} & \multicolumn{3}{c}{ ADM Relative Error } & \multicolumn{3}{c}{ FEM Relative Error } \\
\cline { 2 - 7 } & Four terms & Five terms & Six terms & 50 & 100 & 150 \\
\hline 0.0 & 0.0 & 0.0 & 0.0 & 0.0 & 0.0 & 0.0 \\
0.02 & $0.453 \times 10^{-3}$ & $1.123 \times 10^{-4}$ & $1.013 \times 10^{-4}$ & $0.234 \times 10^{-3}$ & $3.325 \times 10^{-4}$ & $1.120 \times 10^{-4}$ \\
0.04 & $1.987 \times 10^{-4}$ & $1.347 \times 10^{-4}$ & $0.047 \times 10^{-4}$ & $1.126 \times 10^{-3}$ & $1.034 \times 10^{-4}$ & $2.613 \times 10^{-5}$ \\
0.06 & $2.798 \times 10^{-4}$ & $0.340 \times 10^{-4}$ & $0.434 \times 10^{-5}$ & $2.023 \times 10^{-2}$ & $1.122 \times 10^{-2}$ & $1.106 \times 10^{-5}$ \\
0.08 & $1.001 \times 10^{-5}$ & $0.150 \times 10^{-5}$ & $0.504 \times 10^{-6}$ & $4.750 \times 10^{-3}$ & $2.322 \times 10^{-4}$ & $1.457 \times 10^{-6}$ \\
0.10 & $1.338 \times 10^{-5}$ & $2.243 \times 10^{-5}$ & $1.243 \times 10^{-5}$ & $6.445 \times 10^{-3}$ & $7.805 \times 10^{-5}$ & $3.345 \times 10^{-6}$ \\
\hline
\end{tabular}

Table 2. Error obtained using decomposition method for example 2.

\begin{tabular}{ccccccc}
\hline & \multicolumn{3}{c}{ ADM Relative Error } & \multicolumn{3}{c}{ FEM Relative Error } \\
\cline { 2 - 7 } $\mathrm{t}$ & Four terms & Five terms & Six terms & 50 & 100 & 150 \\
\hline 0.0 & 0.0 & 0.0 & 0.0 & 0.0 & 0.0 & 0.0 \\
0.02 & $1.331 \times 10^{-3}$ & $3.408 \times 10^{-5}$ & $1.408 \times 10^{-4}$ & $0.435 \times 10^{-3}$ & $3.025 \times 10^{-4}$ & $1.420 \times 10^{-4}$ \\
0.04 & $9.034 \times 10^{-3}$ & $1.000 \times 10^{-4}$ & $9.00 \times 10^{-4}$ & $1.512 \times 10^{-3}$ & $1.034 \times 10^{-4}$ & $2.152 \times 10^{-5}$ \\
0.06 & $1.141 \times 10^{-3}$ & $1.092 \times 10^{-4}$ & $9.092 \times 10^{-5}$ & $3.023 \times 10^{-2}$ & $0.122 \times 10^{-3}$ & $1.102 \times 10^{-5}$ \\
0.08 & $3.114 \times 10^{-3}$ & $2.843 \times 10^{-4}$ & $2.843 \times 10^{-4}$ & $4.750 \times 10^{-3}$ & $3.421 \times 10^{-5}$ & $1.456 \times 10^{-6}$ \\
0.10 & $7.081 \times 10^{3}$ & $0.774 \times 10^{-4}$ & $0.075 \times 10^{-4}$ & $6.445 \times 10^{-3}$ & $6.505 \times 10^{-5}$ & $3.345 \times 10^{-6}$ \\
\hline
\end{tabular}


Table 3. Error obtained using decomposition method with four five and six terms for example 3.

\begin{tabular}{ccccccc}
\hline & \multicolumn{3}{c}{ Relative Error } & \multicolumn{3}{c}{ Number of elements } \\
\cline { 2 - 6 } $\mathrm{n}$ & Four terms & Five terms & Six terms & 50 & 100 & 150 \\
\hline 0.0 & 0.0 & 0.0 & 0.0 & 0.0 & 0.0 & 0.0 \\
0.02 & $4.335 \times 10^{-2}$ & $3.425 \times 10^{-2}$ & $1.425 \times 10^{-2}$ & $0.335 \times 10^{-3}$ & $3.325 \times 10^{-4}$ & $1.420 \times 10^{-4}$ \\
0.04 & $1.512 \times 10^{-2}$ & $1.034 \times 10^{-2}$ & $1.634 \times 10^{-2}$ & $1.512 \times 10^{-3}$ & $1.034 \times 10^{-4}$ & $2.653 \times 10^{-5}$ \\
0.06 & $3.023 \times 10^{-2}$ & $1.122 \times 10^{-2}$ & $1.002 \times 10^{-2}$ & $3.023 \times 10^{-2}$ & $1.122 \times 10^{-2}$ & $1.102 \times 10^{-5}$ \\
0.08 & $4.750 \times 10^{-3}$ & $2.322 \times 10^{-4}$ & $1.022 \times 10^{-4}$ & $4.750 \times 10^{-3}$ & $2.322 \times 10^{-4}$ & $1.456 \times 10^{-6}$ \\
0.10 & $6.445 \times 10^{-3}$ & $7.805 \times 10^{-4}$ & $2.805 \times 10^{-4}$ & $6.445 \times 10^{-3}$ & $7.805 \times 10^{-5}$ & $3.345 \times 10^{-6}$ \\
\hline
\end{tabular}

Table 4. Error obtained using finite element method example 1.

\begin{tabular}{cccccc}
\hline \multirow{2}{*}{$\begin{array}{c}\text { F.E.M } \\
\text { p version }\end{array}$} & 50 elements & 75 elements & 100 elements & 125 elements & 150 elements \\
\cline { 2 - 5 } & $1.071 \times 10^{-3}$ & $2.138 \times 10^{-3}$ & $6.306 \times 10^{-4}$ & $4.006 \times 10^{-4}$ & $1.303 \times 10^{-5}$ \\
\hline Quadratic & $1.0 . M$ h version & \\
Cubic & $2.402 \times 10^{-4}$ & $1.485 \times 10^{-4}$ & $2.759 \times 10^{-5}$ & $2.051 \times 10^{-5}$ & $2.759 \times 10^{-5}$ \\
Quartic & $1.541 \times 10^{-5}$ & $7.683 \times 10^{-6}$ & $1.481 \times 10^{-6}$ & $1.6211 \times 10^{-6}$ & $0.381 \times 10^{-6}$ \\
\hline
\end{tabular}

Table 5. Error obtained using finite element method example 2.

\begin{tabular}{|c|c|c|c|c|c|}
\hline \multirow{2}{*}{$\begin{array}{c}\text { F.E.M } \\
\text { p version }\end{array}$} & \multicolumn{5}{|c|}{ F.E.M h version } \\
\hline & 100 elements & 125 elements & 150 elements & 175 elements & 200 elements \\
\hline Quadratic & $3.971 \times 10^{-3}$ & $2.338 \times 10^{-3}$ & $7.378 \times 10^{-4}$ & $4.006 \times 10^{-4}$ & $1.303 \times 10^{-5}$ \\
\hline Cubic & $2.502 \times 10^{-4}$ & $1.485 \times 10^{-4}$ & $2.759 \times 10^{-5}$ & $2.051 \times 10^{-5}$ & $2.759 \times 10^{-5}$ \\
\hline Quartic & $1.541 \times 10^{-5}$ & $8.683 \times 10^{-6}$ & $0.683 \times 10^{-6}$ & $1.621 \times 10^{-6}$ & $0.381 \times 10^{-6}$ \\
\hline
\end{tabular}

Table 6. Error obtained using finite element method example 3.

\begin{tabular}{|c|c|c|c|c|c|}
\hline \multirow{2}{*}{$\begin{array}{c}\text { F.E.M } \\
\text { p version }\end{array}$} & \multicolumn{5}{|c|}{ F.E.M h version } \\
\hline & 100 elements & 125 elements & 150 elements & 175 elements & 200 elements \\
\hline Quadratic & $3.271 \times 10^{-3}$ & $2.138 \times 10^{-3}$ & $6.306 \times 10^{-4}$ & $4.006 \times 10^{-4}$ & $1.303 \times 10^{-5}$ \\
\hline Cubic & $2.402 \times 10^{-4}$ & $1.485 \times 10^{-4}$ & $2.759 \times 10^{-5}$ & $2.051 \times 10^{-5}$ & $2.059 \times 10^{-5}$ \\
\hline Quartic & $1.541 \times 10^{-5}$ & $7.683 \times 10^{-6}$ & $1.681 \times 10^{-6}$ & $1.621 \times 10^{-6}$ & $0.331 \times 10^{-7}$ \\
\hline
\end{tabular}

From Tables 4-6, we observe that the $\mathrm{h}$ and the $\mathrm{p}$ collocation FEM method yields a almost exact solution for elements in order $=50,100$, and 150, respectively [14] an investigate the effect of the choice of the number of elements around the singularity points over comes the failed of the traditional ADM on the numerical solution of Troesch's problem. Tables 4-6 provide the relative error obtained by the $h$ and the $\mathrm{p}$ shape-spline method,

We observe generally notable change in the numerical results when the number of elements and the degree increased, we can say almost exact. 
From Tables 1-3, it is evident that the relative error for all given examples decreases as the number of terms of the ADM increase, convergence is rapid and the relative error is small.

Likewise, result in Tables 4-6 shows the F.E.M error as the number of elements is subdivided (the $\mathrm{h}$ version) also as the degree of the polynomial is increased ( $\mathrm{p}$ version). With more dense elements near $t=0$ has the upper hand results.

\section{Conclusion}

In this work, the Adomians decomposition method, and the finite element method $\mathrm{p}$ and the $\mathrm{h}$ versions have been successfully applied to find the solution of nonlinear Emden-Fowler Equation. For general cases, Adomians decomposition method usually fails to solve singular initial value problems of Emden-Fowler type, while an effective modification of FEM that only requires denser elements around the singularity point is introduced. We conclude that FEM is a powerful method for the solution of nonlinear Emden-Fowler Equation. However, the computations by the ADM are simpler and faster than FEM techniques. The FME offers great advantages of straightforward applicability, computational efficiency and high accuracy.

\section{Conflicts of Interest}

The author declares no conflicts of interest regarding the publication of this paper.

\section{References}

[1] James, S. and Wong, W. (1975) On the Generalized Emden-Fowler Equation Society for Industrial and Applied Mathematics. SIAM Review, 17, 339-360. https://doi.org/10.1137/1017036

[2] Adomians, G., Rach, R. and Shawagfeh, N.T. (1995) On the Analytic Solution of the Lane-Emden Equation. Physics Letters, 8, 161-181. https://doi.org/10.1007/BF02187585

[3] Metropolis, N. and Reitz, J.R. (1951) Solutions of the Fermi-Thomas-Dirac Equation. The Journal of Chemical Physics, 19, 555. https://doi.org/10.1063/1.1748292

[4] Adomians, G. (1990) A Review of the Decomposition Method and Some Recent Results for Nonlinear Equations. Mathematical and Computer Modelling, 13, 17-43. https://doi.org/10.1016/0895-7177(90)90125-7

[5] Evans, D.J. and Hossen, K.A. (2000) Asymptotic Decomposition of Mildly Nonlinear Differential Equation. International Journal of Computer Mathematics, 78, 569-573. https://doi.org/10.1080/00207160108805132

[6] Roberts, S.M. and Shipman, J.S. (1976) On the Closed form Solution of Troesch's Problem. Journal of Computational Physics, 21, 291-304. https://doi.org/10.1016/0021-9991(76)90026-7

[7] Scott, M.R. (1975) On the Conversion of Boundary-Value Problems into Stable Initial-Value Problems via Several Invariant Imbedding Algorithms. In: Aziz, A.K., Ed., Numerical Solutions of Boundary-Value Problems for Ordinary Differential 
Equations, Academic Press, New York, 89-146. https://doi.org/10.1016/B978-0-12-068660-5.50008-9

[8] Khuri, S.A. (2003) A Numerical Algorithm for Solving the Troesch's Problem. International Journal of Computer Mathematics, 80, 493-498. https://doi.org/10.1080/0020716022000009228

[9] Feng, X., Mei, L. and He, G. (2007) An Efficient Algorithm for Solving Troesch's Problem. Applied Mathematics and Computation, 189, 500-507. https://doi.org/10.1016/j.amc.2006.11.161

[10] Chang, S.H. and Chang, I.L. (2008) A New Algorithm for Calculating the One-Dimensional Differential Transform of Nonlinear Functions. Applied Mathematics and Computation, 195, 799-808. https://doi.org/10.1016/j.amc.2007.05.026

[11] Chang, S.-H. (2010) A Variational Iteration Method for Solving Troesch's Problem. Journal of Computational and Applied Mathematics, 234, 3043-3047. https://doi.org/10.1016/j.cam.2010.04.018

[12] Deeba, E.Y. and Khuri, S.A. (1996) A Decomposition Method for Solving the Nonlinear Klein-Gordon Equation. Journal of Computational Physics, 124, 442-448. https://doi.org/10.1006/jcph.1996.0071

[13] Adomians, G. (1986) Nonlinear Stochastic Operator Equations. Academic Press, New York. https://doi.org/10.1016/B978-0-12-044375-8.50012-5

[14] Hossen, K.A. (1984) Finite Element Solutions for Elliptic Partial Differential Equations. PhD Thesis, Loughborough University of Technology, Loughborough.

[15] Hossein, J., Tayyebi, E., Sadeghi, S. and Khalique, C.M. (2014) New Modification of the Adomian Decomposition Method for Nonlinear Integral Equations. International Journal of Advances in Applied Mathematics and Mechanics, 1, 33-39.

[16] Almazmumy, M., Hendi, F.A., Bakodah, H.O. and Alzumi, H. (2012) Recent Modifications of Adomian Decomposition Method for Initial Value Problem in Ordinary Differential Equations. American Journal of Computational Mathematics, 2, 228-234. https://doi.org/10.4236/ajcm.2012.23030

[17] Seng, V. and Abbaoui, K.Y. (1996) Adomians for Nonlinear Operators. Mathematical and Computer Modelling, 24, 59-65. https://doi.org/10.1016/0895-7177(96)00080-5

[18] Rosen, R. (1968) Matrix Bandwidth Minimization. Proceedings of 23 rd National Conference, ACM, ACM Publication P-68, Brandon/Systems Press, Princeton, NJ, 585-595. https://doi.org/10.1145/800186.810622

[19] Cuthill, E. and McKee, J. (1969) Reducing the Bandwidth of Sparse Symmetric Matrices. Proceedings 24th National Conference of ACM, August 1969, 157-172. https://doi.org/10.1145/800195.805928

[20] Cheng, K.Y. (1973) Minimizing the Bandwidth of Sparse Symmetric Matrices. Computing, 11, 103-110.

[21] Maplesoft User Case Studies. http://www.maplesoft.com/company/casestudies

[22] Granville, S. (1981) TWODEPEP. International Mathematical and Statistical Libraries. IMSL Statistical Libraries, Inc., Houston. 